\title{
BMJ Open Randomised controlled trial of integrated trauma-focused psychotherapy for traumatic stress and substance use among adolescents: trial protocol
}

\author{
Katherine L Mills (1) , ${ }^{1}$ Emma Barrett, ${ }^{1}$ Sudie E Back, ${ }^{2}$ Vanessa E Cobham, ${ }^{3,4}$ \\ Sarah Bendall, ${ }^{5,6}$ Sean Perrin, ${ }^{7}$ Kathleen T Brady, ${ }^{2}$ Joanne Ross, ${ }^{8}$ Natalie Peach, ${ }^{1}$ \\ Ivana Kihas, ${ }^{1}$ Joanne Cassar, ${ }^{1}$ Olivia Schollar-Root, ${ }^{1}$ Maree Teesson ${ }^{1}$
}

To cite: Mills KL, Barrett E, Back SE, et al. Randomised controlled trial of integrated trauma-focused psychotherapy for traumatic stress and substance use among adolescents: trial protocol. BMJ Open 2020;10:e043742. doi:10.1136/ bmjopen-2020-043742

- Prepublication history and additional material for this paper are available online. To view these files, please visit the journal online (http://dx.doi. org/10.1136/bmjopen-2020043742).

Received 12 August 2020 Revised 30 0ctober 2020 Accepted 16 November 2020

Check for updates

(C) Author(s) (or their employer(s)) 2020. Re-use permitted under CC BY-NC. No commercial re-use. See rights and permissions. Published by BMJ.

For numbered affiliations see end of article.

Correspondence to

Professor Katherine L Mills;

katherine.mills@sydney.edu.au

\section{ABSTRACT}

Introduction Post-traumatic stress disorder (PTSD) and substance use disorder frequently co-occur and tend to have their onset during adolescence. Although research has highlighted the importance of treating these disorders in an integrated fashion, there is a dearth of empirically validated integrated treatment options for adolescents with this comorbidity. This paper describes the study protocol for a randomised controlled trial (RCT) examining the efficacy of an integrated trauma-focused cognitivebehavioural treatment for traumatic stress and substance use among adolescents (Concurrent Treatment of PTSD and Substance Use Using Prolonged Exposure - Adolescent (COPE-A)), relative to a supportive counselling control condition (Person-Centred Therapy (PCT)).

Methods and analysis A two-arm, parallel, singleblind RCT with blinded follow-up at 4 and 12 months poststudy entry will be conducted in Sydney, Australia. Participants ( $\mathrm{n}$ 100 adolescents aged 12-18 years) and their caregivers (caregiver participation is optional) will be allocated to undergo either COPE-A or PCT (allocation ratio 1:1) using minimisation. Both therapies will be delivered individually by project psychologists over a maximum of 16 sessions of 60-90 min duration and will include provision of up to four $30 \mathrm{~min}$ optional caregiver sessions. The primary outcome will be between-group differences in change in the severity of PTSD symptoms from baseline to 4-month follow-up, as measured by the ClinicianAdministered PTSD Scale for Children and Adolescents for DSM-5.

Ethics and dissemination Ethical approval has been obtained from the human research ethics committees of the Sydney Children's Hospital Network (HREC/17/ SCHN/306) and the University of Sydney (HREC 2018/863). Findings will be published in peer-reviewed journals and presented at scientific conferences.

Trial registration number ACTRN12618000785202;

Pre-reults.

Protocol version Version 1, 31 July 2017.

\section{INTRODUCTION}

Evidence from around the world has brought to light alarmingly high rates of exposure to potentially traumatic events (PTEs) and, in
Strength and limitations of this study

- Using a rigorous randomised controlled trial design, this study will provide one of the first examinations of integrated trauma-focused psychotherapy for traumatic stress and substance use among adolescents aged $12-18$ years.

- The use of manualised supportive counselling as an active control group represents an approximation of current practice with the added rigour of standardised delivery.

- Comprehensive collection of data by interviewers blind to group allocation will allow for a detailed examination of factors associated with this comorbidity and their impact on treatment outcomes.

- Feasibility and generalisability will be optimised using a multimethod recruitment strategy.

- The hidden nature of these conditions, both characterised by avoidance, may limit clinician referrals and self-referrals and impact on recruitment.

many cases, repeated exposure among children and adolescents. ${ }^{1-3}$ These PTEs include a wide range of terrifying and life-threatening experiences, commonly physical and sexual assault, witnessing violence, accidents and natural disasters. A review of 32 studies conducted across 13 countries concluded that rates of exposure to PTEs peak in adolescence, with up to $80 \%$ having been exposed to at least one PTE and 14\% meeting criteria for a diagnosis of post-traumatic stress disorder (PTSD). ${ }^{3}$

A strong and consistent relationship has been found between child and adolescent exposure to PTE and the development of subsequent alcohol and other drug problems. ${ }^{4}$ Approximately one-half of adolescents suffering from PTSD also suffer from a co-occurring substance use disorder (SUD). ${ }^{3}$ Studies have found that adolescents with PTSD tend to use substances to relieve their 
symptoms of PTSD (ie, 'self-medication'). ${ }^{45}$ Furthermore, early life exposure to PTEs has been found to have longlasting effects, not only on the stress response system but also on neurotransmitter systems involved in the positive reinforcing effects of alcohol and other drugs, increasing vulnerability to the development of SUDs. ${ }^{4}$

Studies of adolescent samples have found that in $62 \%-75 \%$ of cases, the onset of PTSD occurs prior to SUD. ${ }^{67}$ However, intoxication and high-risk behaviours associated with substance misuse also place adolescents at increased risk of exposure to further PTEs, such as assault and serious accidents. ${ }^{68}$ Thus, a cyclical relationship can develop, where PTSD symptoms trigger substance misuse, which in turn increases the risk of future PTEs. ${ }^{5}$ Regardless of how both conditions come to co-occur, once established, both disorders serve to maintain and exacerbate the other, ${ }^{5910}$ leading to a chronic course of illness and significant treatment complications. ${ }^{11} 12$

Adolescents who develop both conditions have been found to exhibit significantly greater internalising and externalising problems, including academic and vocational impairment, anxiety, depression, suicidality, poorer physical health, family and social dysfunction, aggression and criminal behaviour. ${ }^{13-15}$ These abnormalities may persist well into adulthood and are linked to increased risk of morbidity and mortality. ${ }^{16}$ Of great concern is the strong association between trauma, substance use and suicide. ${ }^{1417}$ Furthermore, the earlier the onset of PTSD and SUD, the more severe and chronic the illness course for both disorders is likely to be. ${ }^{17-19}$ The earlier effective treatment can be delivered, the more likely it is that this trajectory can be interrupted.

Evidence-based psychological approaches for the treatment of adolescents with PTSD and SUD are, however, lacking. Due to anecdotal concerns regarding the ability of clients with SUD to cope with distressing emotions (and concerns about the potential for deterioration, overdose, suicide and self-harm), historically treatment guidelines recommended that PTSD treatment commence only after a substantial period of abstinence has been achieved. However, PTSD symptoms tend to intensify when a young person is no longer drinking or using other substances, placing them at increased risk of relapse. ${ }^{11}{ }^{20}$ Moreover, studies examining the temporal sequencing of changes in PTSD and SUD symptoms indicate that PTSD should be addressed in order to achieve lasting improvements in substance use outcomes. ${ }^{11} 2021$

Based on these findings, experts and clinical practice guidelines now advocate for the integrated treatment of PTSD and SUD; that is, treatment of both disorders at the same time by the same clinician. ${ }^{22}{ }^{23}$ This integrated approach allows for PTSD to be treated in a way that is tolerable while at the same time addressing the person's substance use. There is, however, a dearth of empirically validated integrated treatment options for adolescents with PTSD and SUD, with only four pilot studies ${ }^{24-27}$ and one larger randomised controlled trial (RCT) $(n=124)^{28}$ conducted to date. Notably, despite promising findings from trials conducted among adults, ${ }^{29}$ none of the therapies examined to date have incorporated prolonged exposure, a gold-standard PTSD treatment.

Concurrent Treatment of PTSD and Substance Use Disorders Using Prolonged Exposure (COPE) is the only published integrated treatment that incorporates goldstandard treatments for PTSD and SUD (ie, cognitivebehavioural therapies that include prolonged exposure). It is designed to be used with adults with any SUD and any type of trauma. ${ }^{30}$ The efficacy of COPE was demonstrated among 103 Australian adults (aged 18+ years) in an RCT. ${ }^{23}$ Despite having a severe clinical profile characterised by extensive histories of trauma and polydrug use, elevated levels of impairment and high rates of psychopathology, participants randomised to undergo COPE demonstrated greater reductions in PTSD symptom severity compared with those randomised to receive usual care. Furthermore, those in the treatment group were less likely to have a PTSD diagnosis at follow-up (56\% vs $79 \%$ ). Secondary analysis found that reductions in PTSD symptomology were associated with significant reductions in severity of dependence, and participants expressed high levels of satisfaction with the treatment. Positive findings have also been found in subsequent trials conducted among adults in the USA ${ }^{31}$;2 ; however, these findings cannot be extrapolated to persons aged under 18 years. Practices supported for adults may not be supported in younger age groups. ${ }^{33}$ Adolescents are not 'little adults', ${ }^{17}$ and in order to be effective, adolescent programmes must be tailored to meet their unique developmental needs. ${ }^{22} 34$ In this trial, we propose to conduct an RCT of a modified version of the COPE intervention tailored specifically to the needs of adolescents aged 12-18 years (COPE-A), relative to a supportive counselling control condition (Person-Centred Therapy (PCT)). It is hypothesised that: 1. adolescents randomised to undergo COPE-A will demonstrate greater pretreatment to post-treatment reductions in PTSD symptom severity compared with adolescents randomised to undergo PCT (primary hypothesis);

2. adolescents randomised to undergo COPE-A will demonstrate reductions in substance use, and improvements in behavioural, physical, emotional, social and educational functioning (secondary hypothesis); and

3. changes observed from pretreatment to post-treatment will be maintained 12 months postbaseline (secondary hypothesis).

\section{METHODS}

\section{Trial design}

This RCT is a parallel group, two-arm superiority trial using patient-level randomisation. Approximately 100 adolescents aged 12-18 years (and their caregivers, if appropriate) will be recruited and randomised to the treatment or control condition (allocation ratio 1:1). Participant flow through the study is depicted in figure 1. 
Figure 1: Flow of participants

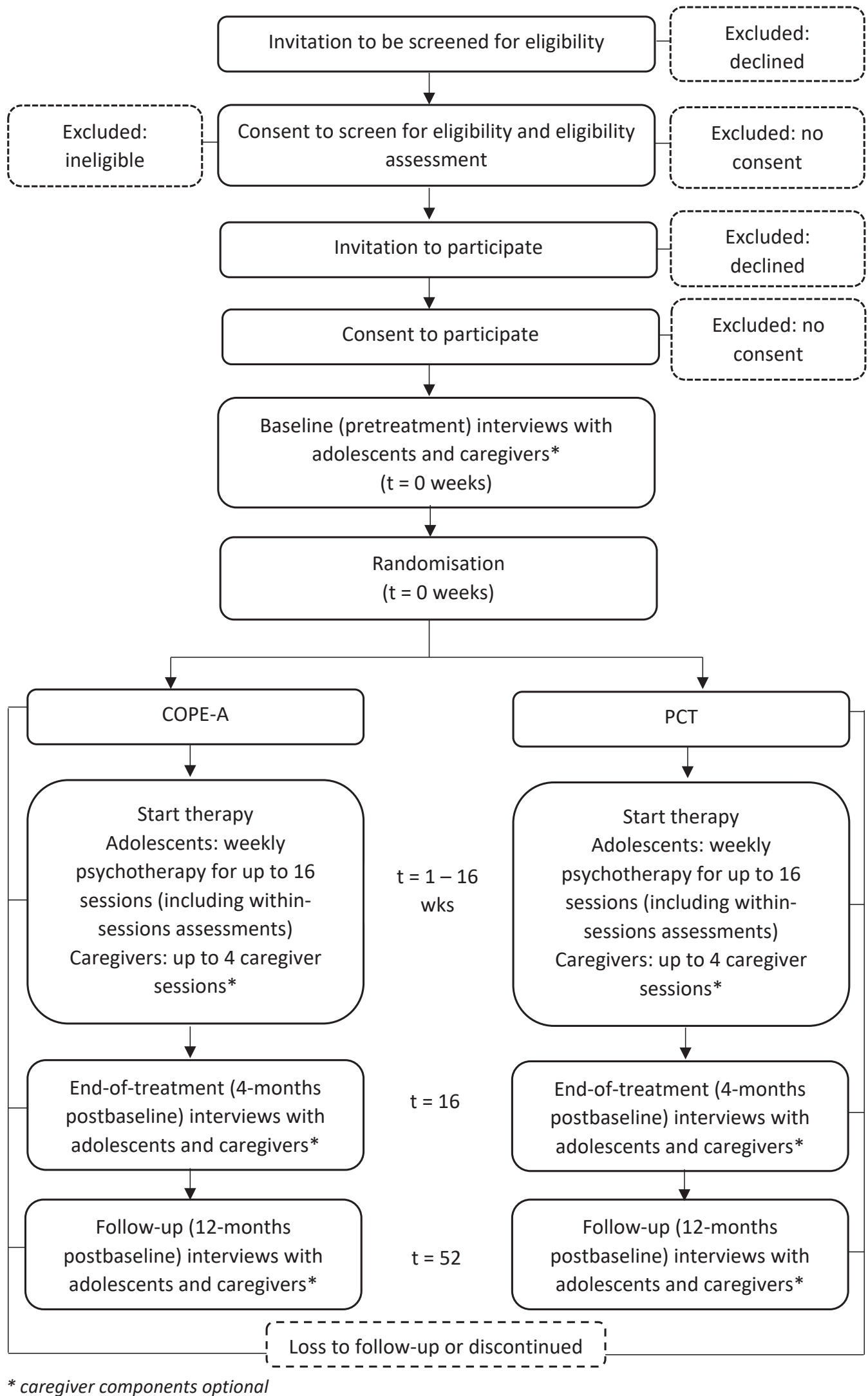

Figure 1 Flow of participants. COPE-A, Concurrent Treatment of PTSD and Substance Use Using Prolonged Exposure Adolescent. PCT, Person-Centred Therapy. 
WHO trial registration data set information is provided in the online supplemental material.

\section{Participants}

Patient and public involvement

Members of the Matilda Centre and National Health and Medical Research Council Centre of Research Excellence in the Prevention and Early Intervention of Mental Illness and Substance Use (PREMISE) Youth Advisory Board (aged 16-25 years) were consulted and provided valuable input towards our recruitment strategy.

\section{Recruitment setting}

Participants will be recruited from Sydney, Australia, using a multimethod recruitment strategy. Referrals will be sought from health, social and welfare services in the government, non-government and private sectors. Online (eg, social media) and offline (eg, radio and print media) advertising targeted towards adolescents, parents/families, teachers, and health and social workers who may be working with the target population will also be implemented. Recruitment commenced on 21 May 2018. It is anticipated that the trial will be completed by June 2022 .

\section{Eligibility criteria}

\section{Inclusion criteria}

Included (1) age 12-18 years; (2) alcohol or other drug use in the past month; (3) history of problematic alcohol or other drug use (cut-off score of 2 on the CRAFFT ${ }^{35}$ ); (4) endorsement of lifetime exposure to at least one PTE according to the University of California, Los Angeles (UCLA) Child/Adolescent Post-traumatic Stress Disorder Reaction Index for DSM-5 (PTSD-RI); ${ }^{36}$ (5) meeting DSM-5 criteria for a subthreshold or full diagnosis of current PTSD (ie, past month) according to the PTSD-RI (subthreshold diagnosis defined as meeting criteria A; endorsing a minimum of one symptom for criteria B, C, $\mathrm{D}$ and $\mathrm{E}$; and meeting criteria $\mathrm{F}$ and $\mathrm{G}$ ); and (6) fluency in English.

\section{Exclusion criteria}

Included (1) recent history of attempted suicide (ie, within the past 3 months) or current risk of suicide or serious self-harm; (2) current symptoms of psychosis based on the Mini International Neuropsychiatric Interview for Children and Adolescents (score of $>1$ ) ${ }^{37}$ and clinical observation; (3) cognitive impairment severe enough to impede treatment based on clinical observation, and (4) ongoing trauma-related threat or ongoing unsupervised contact with the alleged perpetrator.

There will be no limitations on participants' ability to receive concomitant care or other interventions during the trial.

\section{Interventions}

Participating adolescents will be randomised to undergo either COPE-A or PCT. Participants in both arms may undergo up to 16 individual sessions of $60-90 \mathrm{~min}$ with a registered psychologist employed on the trial. Both therapies include provision of an optional caregiver component. The caregiver components for both therapies consist of up to four $30 \mathrm{~min}$ sessions with the adolescent's psychologist, with caregiver defined as a parent, guardian, relative or other responsible adult from whom the adolescent may seek support. The caregiver components provide psychoeducation about substance use and trauma responses, information about the treatment the adolescent will undergo and how the caregiver can best support the adolescent through therapy.

Psychologists employed on the project will be trained in and will deliver both therapies. All sessions will be audio recorded and reviewed weekly by the project coordinator using a checklist to document compliance, and $10 \%$ of each therapist's sessions will be randomly selected and rated for compliance by a blind independent assessor.

Concurrent Treatment of PTSD and Substance Use Using Prolonged Exposure-Adolescent version (COPE-A)

The components of the COPE-A programme include psychoeducation, motivational interviewing, cognitivebehavioural therapy (CBT) for substance use and traumafocused CBT for PTSD (including imaginal and in vivo prolonged exposure). The COPE-A programme is based on the adult COPE therapy, ${ }^{30}$ which has been modified to meet the developmental needs of adolescents. While the core elements have not changed, the rationale for prolonged exposure, the metaphors and examples used, and the manner in which the imaginal exercises are constructed have been modified to be more developmentally appropriate (eg, simplified rationale, illustrative metaphors included). There is also a stronger emphasis on motivational interviewing (to increase participant engagement and commitment to change) and emotion regulation to provide skills in building relationships, avoiding high-risk situations, and using coping strategies that can decrease risk of trauma exposure and increase resilience.

\section{Person-Centred Therapy (PCT)}

PCT is an active therapy that has been shown to produce significant improvements in PTSD and substance use..$^{38} 39$ PCT incorporates three principles which have been identified as non-specific factors of all successful psychotherapies: genuineness or congruence, unconditional positive regard and accurate empathy. ${ }^{39}$ Therapists provide active listening, empathy and encouragement to talk about feelings and to express belief in the participant's ability to cope. Sessions are structured around the premise of 'invite the client to talk using the techniques of genuineness, unconditional positive regard and accurate empathy'. Participants choose when, how, and whether or not to discuss their trauma. The use of this form of manualised supportive counselling as an active control group represents an approximation of current practice with the added rigour of standardised delivery. 


\section{Outcomes}

\section{Primary outcomes}

Between-group differences in change in the severity of PTSD symptoms (Clinician-Administered PTSD Scale for Children and Adolescents for DSM-5 (CAPS-CA-5) scores $)^{40}$ from baseline to 4 months postbaseline (ie, endof-treatment) follow-up.

\section{Secondary outcomes}

Between-group differences in change in relation to the following variables from baseline to 4 months postbaseline:

- Proportion of participants remitted from subthreshold/full PTSD.

- Percentage of days using substances (measured using timeline followback (TLFB) ${ }^{41}$ ).

- Number of dependence criteria met (Diagnostic Interview Schedule for Children (DISC-5) ${ }^{42}$ ).

- Self-confidence to resist the urge to use substances (Brief Situational Confidence Questionnaire (BSCQ) scores $\left.^{43}\right)$.

- Post-traumatic cognitions (Child Post-traumatic Cognitions Inventory scores (CPTCI ${ }^{44}$ ).

- Physical, emotional, social and educational functioning (Paediatric Quality of Life Child and Parent Version scores (PedsQL-C and PedsQL-P, respectively) ${ }^{45}$ ).

Between-group differences in changes observed relating to the primary and secondary outcomes listed previously from 4 to 12 months postbaseline will also be examined.

\section{Other outcomes}

At 4 and 12 months postbaseline: anxiety and depression (Revised Children's Anxiety and Depression Scale $\left.{ }^{46}\right)$; suicide and self-harm; ability to manage emotions (Emotion Regulation Questionnaire ${ }^{47}$ ); distress tolerance (Distress Tolerance Scale $^{47}$ ); perseverative thinking (Perseverative Thinking Questionnaire ${ }^{48}$ ); selfcompassion (Self-Compassion Scale-Short Form ${ }^{49}$ ); behaviour (Strengths and Difficulties Questionnaire, ${ }^{50}$ Aggression Questionnaire, derived from the Young Minds Matter interview, ${ }^{51}$ and Opiate Treatment Index ${ }^{52}$ ); disordered eating (Youth Eating Disorder Examination Questionnaire ${ }^{53}$ ); health service use; family functioning (Family Assessment Device ${ }^{54}$ ); client satisfaction (Youth Client Satisfaction Questionnaire (Y-CSQ) ${ }^{55}$ and Clinet Satisfaction Questionnaire $\left.\left(\mathrm{CSQ}^{56}\right)\right)$; and adverse events. Between-session changes in PTSD symptom severity (PTSD-RI) and between-session and within-session changes in craving (measured on a Likert scale) will also be examined.

\section{Data collection}

Adolescents and participating caregivers will undergo three structured interviews with the project's research officers (who will be blind to treatment allocation) at baseline (study entry), and at 4 and 12 months postbaseline. The instruments included in these interviews (tables 1 and 2) measure the primary and secondary outcomes of the study, hypothesised moderators and mediators of outcome, and potential confounders. Adolescents will also complete brief questionnaires in each session to monitor PTSD symptom change (PTSD-RI), substance use (TLFB and craving scale) and suicide risk (Suicide Behaviors Questionnaire-Revised). ${ }^{57}$ The project psychologists will keep a log of adolescent and caregiver session attendance. Measures of client satisfaction (Y-CSQ and CSQ) and adolescent and therapist therapeutic alliance (Working Alliance Inventory-Short Revised ${ }^{58}$ ) will be completed at treatment completion.

\section{Data management}

Data will be collected using structured hard-copy (paperand-pencil) interviews and entered into SPSS data files. Separate files will be maintained for the (1) adolescent and (2) caregiver interview, (3) within session and therapist measures, and (4) treatment credibility, client satisfaction and therapeutic alliance. Adolescent and caregiver interview data will be entered by the project's research officers; within session and therapist measures will be entered by the project's psychologists; client satisfaction and therapeutic alliance measures will be entered by the project coordinator. To maximise accuracy and consistency of data entry, a data dictionary will be used to define the variables and to specify how they are to be coded. Data verification activities (including value range and consistency checks to detect missing or erroneously entered data) will be performed on $100 \%$ of the data and erroneous entries will be corrected.

All hard-copy materials will be stored in locked filing cabinets at the Matilda Centre, University of Sydney, with access restricted to authorised personnel. All electronic materials will be stored on a secure server hosted by the University of Sydney and will be password protected. All records that contain names or other personal identifiers (such as locator forms and consent forms) will be stored separately from data collected from interviews and within sessions; interview and session materials will be identified by a participant identification number.

Interim analyses will be performed to determine whether any adjustments are needed to the trial design, such as sample size re-estimation based on interim between-group effect sizes, or changes to the selection criteria. As one of the first studies of its kind, futility analysis will not be conducted. The trial will only be stopped if it is deemed unsafe to continue by the trial investigators and/or the human research ethics committees (HRECs) that have approved the trial. Data and safety monitoring will be undertaken by the trial investigators who will be unblinded to allocation.

\section{Adverse events}

Adverse events will be monitored and reported according to the trial's adverse events protocol (available from the authors on request) which documents procedures for identifying, assessing, documenting and, where relevant, reporting to authorities. An adverse event in this trial 
Table 1 Content of adolescent interviews*

\begin{tabular}{|c|c|c|c|c|}
\hline Domain & Measure & Baseline & 4-month follow-up & 12-month follow-up \\
\hline Demographics & Study derived & $x$ & $x$ & $x$ \\
\hline Substance use & TLFB $^{41}$ & $x$ & $x$ & $x$ \\
\hline Substance use disorder & DISC- $5^{42}$ & $x$ & $x$ & $x$ \\
\hline Substance use motives & $\begin{array}{l}\text { Drinking Motives Questionnaire - } \\
\text { Revised (DMQ-R) }\end{array}$ & $x$ & $x$ & $x$ \\
\hline Resisting substance use & $\mathrm{BSCQ}^{43}$ & $x$ & $x$ & $x$ \\
\hline Exposure to PTE & PTSD-RI ${ }^{36}$ & $x$ & $x$ & $x$ \\
\hline $\begin{array}{l}\text { Adverse childhood } \\
\text { experiences }\end{array}$ & $\begin{array}{l}\text { Adverse Childhood Experiences } \\
\text { (ACE) scale }{ }^{60}\end{array}$ & $x$ & $x$ & $x$ \\
\hline PTSD & CAPS-CA- $5^{40}$ & $x$ & $x$ & $x$ \\
\hline Post traumatic cognitions & $\mathrm{CPTCl}^{44}$ & $x$ & $x$ & $x$ \\
\hline Service use & Adapted from $\mathrm{YMM}^{51}$ & $x$ & $x$ & $x$ \\
\hline Family functioning & $\mathrm{FAD}^{54}$ & $x$ & $x$ & $x$ \\
\hline Quality of life & PedsQL-C ${ }^{61}$ & $x$ & $x$ & $x$ \\
\hline Functioning & Youth Self-Report (YSR) scale ${ }^{62}$ & $x$ & $x$ & $x$ \\
\hline Depression and anxiety & $\operatorname{RCADS}^{46}$ & $x$ & $x$ & $\mathrm{x}$ \\
\hline Suicide and self-harm & Adapted from $\mathrm{YMM}^{51} / \mathrm{SBQ}-\mathrm{R}^{57}$ & $x$ & $x$ & $x$ \\
\hline Perseverative thinking & PTQ-C ${ }^{48}$ & $x$ & $x$ & $x$ \\
\hline Behaviour & $\mathrm{SDQ}^{50}$ & $x$ & $x$ & $x$ \\
\hline Aggression & Adapted from $\mathrm{YMM}^{51}$ and $\mathrm{OT}{ }^{52}$ & $x$ & $x$ & $x$ \\
\hline Bullying & $\begin{array}{l}\text { Adapted from the Olweus Bullying } \\
\text { Questionnaire }^{63}\end{array}$ & $x$ & $x$ & $x$ \\
\hline Personality & $\begin{array}{l}\text { Ten Item Personality Inventory } \\
(\mathrm{TIPI})^{64}\end{array}$ & $x$ & $x$ & $x$ \\
\hline Self-compassion & SCS-SF ${ }^{49}$ & $x$ & $x$ & $x$ \\
\hline Eating behaviours & YEDE-Q ${ }^{53}$ & $x$ & $x$ & $x$ \\
\hline Emotion regulation & $E R Q-C A^{47}$ & $x$ & $x$ & $\mathrm{x}$ \\
\hline Distress tolerance & DTS $^{65}$ & $x$ & $x$ & $\mathrm{x}$ \\
\hline Adverse events & Study derived & - & $x$ & $x$ \\
\hline Client satisfaction & $\begin{array}{l}\text { Youth Client Satisfaction } \\
\text { Questionnaire }^{55}\end{array}$ & - & $x$ & - \\
\hline Therapeutic alliance & WAI-SR ${ }^{58}$ & - & $x$ & - \\
\hline
\end{tabular}

*All instruments administered by the project's research officers blind to group allocation, with the exception of client satisfaction and therapeutic alliance. These were administered by the study project coordinator.

BSCQ, Brief Situational Confidence Questionnaire; CAPS-CA-5, Clinician-Administered PTSD Scale for Children and Adolescents for DSM5; CPTCI, Child Post-traumatic Cognitions Inventory; DISC-5, Diagnostic Interview Schedule for Children; DTS, Distress Tolerance Scale; ERQ-CA, Emotion Regulation Questionnaire; FAD, Family Assessment Device; OTI, Opiate Treatment Index; PedsQL-C, Paediatric Quality of Life Child Version; PTQ-C, Perseverative Thinking Questionnaire-Child Version; PTSD-RI, Post-traumatic Stress Disorder Reaction Index for DSM-5; RCADS, Revised Children's Anxiety and Depression Scale; SBQ-R, Suicide Behaviors Questionnaire-Revised; SCS-SF, Self-Compassion Scale-Short Form; SDQ, Strengths and Difficulties Questionnaire; TLFB, timeline followback; WAI-SR, Working Alliance Inventory-Short Revised; YEDE-Q, Youth Eating Disorder Examination Questionnaire; YMM, Young Minds Matter.

refers to an untoward occurrence during the trial, which may or may not be causally related to the intervention or other aspects of trial participation, that occurs after entry into the study (ie, after the participant has provided written consent) through to the 12-month follow-up interview. Adverse events will be coded as mild, moderate or severe.
A serious adverse event ( $\mathrm{SAE}$ ) for this study is any untoward occurrence that may result in (or place the participant in imminent risk of) death, serious injury/ harm, severe or permanent disability, or unexpected/ unplanned hospitalisation; or where a participant's actions place someone else at imminent risk of serious harm. Potential adverse events include arrest, runaway, 
Table 2 Content of caregiver interviews*

\begin{tabular}{|c|c|c|c|c|}
\hline Domain & Measure & Baseline & 4-month follow-up & 12-month follow-up \\
\hline Demographics & Study derived & $x$ & $x$ & $x$ \\
\hline Family functioning & $\mathrm{FAD}^{54}$ & $x$ & $x$ & $x$ \\
\hline Adolescent functioning & $\begin{array}{l}\text { Child Behaviour } \\
\text { Checklist } \\
(\mathrm{CBCL})^{62}\end{array}$ & $x$ & $x$ & $x$ \\
\hline Adolescent behaviour & $\mathrm{SDQ}^{50}$ & $x$ & $x$ & $x$ \\
\hline Adverse events & Study derived & - & $\mathrm{X}$ & $x$ \\
\hline Client satisfaction & $\begin{array}{l}\text { Client Satisfaction } \\
\text { Questionnaire } \\
(\mathrm{CSQ}-8)^{56}\end{array}$ & - & $x$ & - \\
\hline
\end{tabular}

*All instruments were administered by the project's research officers blinded to group allocation, with the exception of client satisfaction and therapeutic alliance. These were administered by the study project coordinator.

FAD, Family Assessment Device; PedsQL-P, Paediatric Quality of Life Parent Version; RCADS, Revised Children's Anxiety and Depression Scale; SDQ, Strengths and Difficulties Questionnaire; WAI-SR, Working Alliance Inventory-Short Revised.

unexpected homelessness, school suspension/expulsion/dropout, non-serious physical assault and clinically significant deterioration of illness. Potential SAEs include exposure to life-threatening violence (victim/witness), sexual assault/abuse, suicide, serious self-harm, violent/ homicidal behaviour, hospitalisation and death.

Adverse events will be identified via the Adverse Events Questionnaire (administered as part of the interviews of 4 and 12 months), within-session measures, reports made to clinicians during the context of therapy sessions and spontaneous reports made to any members of the project team at any stage of the study. Adverse events and SAEs will be classified as being either expected/anticipated or unexpected/unanticipated, study-related or non-studyrelated. Study-related events will be further classified as being either related to the therapy or to other aspects of the trial protocols and procedures. Determination of relatedness of an event to the study therapy will be based on a temporal relationship to the therapy sessions, whether the event is unexpected or unexplained, given the participant's clinical course, previous medical conditions, and concomitant medications or interventions. SAEs considered to be study-related will be reported to relevant HRECs.

\section{Randomisation}

Intervention allocation will be undertaken independent of the study by the National Health and Medical Research Council Clinical Trials Centre following the baseline interview. To ensure balance between the groups, a process of minimisation will be used with allocation according to sex (male vs female), age (12-14vs 15-18years), severity of SUD (mild: 2-3 criteria met, moderate: $4-5$ criteria met, severe: $\geq 6$ criteria met), trauma type (single incident vs multiple/prolonged incident) and initial PTSD symptom severity (subthreshold vs full PTSD criteria). Due to the nature of the interventions, participants (both adolescents and caregivers) and the project psychologists cannot be blinded to allocation. Research officers will only be unblinded to a participant's allocation in cases where it was deemed necessary to ensure the participant's or another person's safety.

\section{Sample size}

Sample size calculations were conducted using PASS V.11 to determine the number of participants needed to detect clinically significant change in the primary outcome variable. A sample of $n=74$ ( 37 per group) would provide $80 \%$ power of detecting a 10-point difference between groups in CAPS-CA-5 scores $(\mathrm{SD}=15)$ at 4 months postbaseline at $\alpha=0.05$. The sample size of 74 was inflated by $10 \%$ to accommodate the estimated variance attributable to variables used in the minimisation process $(\mathrm{n}=80)$ and was further adjusted to account for an expected $20 \%$ dropout rate. Hence, the desired sample size is $n=100(n=50$ per group).

\section{Sample retention}

Participating adolescents and caregivers will receive a voucher for completion of each of the research interviews ( $\$ \mathrm{~A} 40$ and $\$ \mathrm{~A} 20$ for adolescents and caregivers, respectively). Comprehensive locator information will be collected at baseline and updated at each follow-up interview. Once enrolled in the study, every reasonable effort will be made to follow up participating adolescents and their caregivers. Interviews will be scheduled at times and locations convenient to the participant, and confirmed either on the day before or on the day of the scheduled appointment time. Participants who discontinue treatment will be retained in the study and will continue to 
be followed up. Participants may withdraw from the study for any reason at any time. Participants will only be withdrawn from the study by the investigators if continued participation is deemed to place either the participant or others at risk of harm. Reasons for loss to follow-up will be documented and reported.

\section{Statistical analysis}

Intention-to-treat analyses will be conducted for all outcome analyses. The trial hypotheses will be tested using a series of generalised estimating equations (GEE) for linear, binomial and Poisson (or negative binomial) distributions. All GEE analyses will use an exchangeable correlation matrix. The end point for the treatment outcome analyses is the 4 months postbaseline (hypotheses 1 and 2); however, outcomes will be examined through to 12 months postbaseline to determine the durability of effects (hypothesis 3). Within-treatment change in PTSD symptom severity and substance use will be examined using GEE or, if there are sufficient data points, group-based trajectory models.

Regression analyses will be used to examine differences between groups in client satisfaction and therapeutic alliance and factors associated with these measures. Adverse events and SAEs will be reported descriptively. Where there are sufficient numbers, between-group differences will be analysed inferentially.

Missing value analysis will be conducted to determine the pattern of missing data. Imputation of missing values and sensitivity analyses will be undertaken as appropriate, depending on the findings of the missing value analysis. Subgroup analyses will be performed based on baseline PTSD symptom severity (subthreshold vs full diagnosis). Exploratory analysis will be undertaken to examine the potential moderating and mediating roles of time-variant and time-invariant factors.

\section{ETHICS AND DISSEMINATION \\ Ethics}

Ethical approval has been obtained from the HRECs of the Sydney Children's Hospital Network (HREC/17/ SCHN/306) and the University of Sydney (HREC 2018/863), and proposed amendments to the study protocol will be reviewed by these committees.

Written informed consent will be sought from all adolescents who will agree to participate in the trial. In keeping with the NSW Health Supplement to the National Statement on Ethical Conduct in Human Research and NSW Policy Directive PD2005_406, requirements pertaining to parental/legal guardian consent will vary, depending on the adolescents' age and maturity:

- Aged 12-13 years: parental/legal guardian consent will be required.

- Aged 14-15 years: parental/legal guardian consent will be sought unless the adolescent objects. In these situations, provided the adolescent is deemed to be a 'mature minor' (ie, they have sufficient understanding and intelligence to enable him/her to understand fully what is proposed), adolescent consent alone will suffice. Adolescents will be assessed for their capacity to consent as a mature minor using guidelines provided by in the Australian Psychological Society.

- Aged 16-18 years: parental/legal guardian consent will not be sought.

Adolescents (and their parents/legal guardians, where involved) will be provided with a verbal explanation of the study and a participant information and consent form (PICF) by either the project's research officers, psychologists or the project coordinator. With the adolescent's consent, we will invite a caregiver to take part in the optional caregiver component. This person does not have to be the adolescent's parent/legal guardian; adolescents may opt for us to invite another responsible adult they are close to; however, if the adolescent is aged 12-13 years, or aged 14-15 years but not considered to be a mature minor, consent to invite a caregiver who is not a parent/legal guardian will first be obtained from his/her parent/legal guardian. The nominated caregiver will be provided with a verbal explanation of what their participation would involve and a PICF.

All hard-copy and electronic materials will be stored securely with access restricted to authorised personnel. All records that contain names or other personal identifiers (such as locator forms and consent forms) will be stored separately from data collected in interviews or within sessions. Interview and within-session materials will be identified by a participant identification number. Hardcopy and electronic data will be retained for a minimum of 15 years following the completion of the study or until the youngest child turns 25, whichever is the longest. Participants' study information will not be released to anyone external to the study without the written permission of the participant, except as required by law.

\section{Dissemination}

Study findings will be disseminated to a range of audiences, including researchers, clinicians and other healthcare providers, policy makers and other key stakeholders, as well as the general community. Multiple methods will be used, including academic publications (peer-reviewed journal articles) and professional newsletters; presentations at scientific meetings, professional workshops, seminars and webinars; and online and social media posts. There are no restrictions placed on publication by the funding body. Authorship on study outputs will be based on the International Committee of Medical Journal Editors recommendations for the conduct, reporting, editing and publication of scholarly work in medical journals.

\section{Author affiliations}

${ }^{1}$ The Matilda Centre for Research in Mental Health and Substance Use, The University of Sydney, Sydney, New South Wales, Australia

${ }^{2}$ Department of Psychiatry and Behavioral Sciences, Medical University of South Carolina, Charleston, South Carolina, USA

${ }^{3}$ School of Psychology, The University of Queensland, Saint Lucia, Queensland, Australia 
${ }^{4}$ Children's Health Queensland, Child and Youth Mental Health Service, Brisbane, Queensland, Australia

${ }^{5}$ Orygen, Parkville, Victoria, Australia

${ }^{6}$ Centre for Youth Mental Health, University of Melbourne, Melbourne, Victoria,

Australia

${ }^{7}$ Department of Psychology, Lund University, Lund, Sweden

${ }^{8}$ National Drug and Alcohol Research Centre, University of New South Wales,

Sydney, New South Wales, Australia

\section{Twitter Sean Perrin @Sean_Perrin}

Contributors KM, EB, SB, VC, SEB, SP, KTB, JR and MT contributed significantly to the planning, conceptualisation, design and successful funding of the trial. NP, IK, $\mathrm{JC}$ and OS-R contributed to obtaining ethical and governance approvals, and the acquisition, analysis and interpretation of data. KM, IK and NP drafted the work; EB SEB, VC, SB, SP, KTB, JR, JC, OS-R and MT provided critical intellectual input into the content of the paper. KM, EB, SEB, VC, SB, SP, KTB, JR, NP, IK, JC, OS-R and MT approved the final published version and agreed to be accountable for all aspects of the work.

Funding This randomised controlled trial was funded by an Australian National Health and Medical Research Council (NHMRC project grant APP1127141) and received support from the NHMRC Centre of Research Excellence in the Prevention and Early Intervention in Mental IIIness and Substance Use (APP1134909). The funders had no role with regard to the study design; collection, management, analysis and interpretation of data; writing of the report; and the decision to submit the report for publication, including whether they will have ultimate authority over any of these activities.

Competing interests $\mathrm{KM}$ is a recipient of grant funding and commissions from the National Health and Medical Research Council (NHMRC), Australian Government, NSW Health, Australian Rotary Health, Central Eastern Sydney Primary Health Network, the Sax Institute, Paul Ramsay Foundation, icare; receives royalties from Oxford University Press for the sale of the adult COPE manual; receives honorarium and payment for lectures, seminars and webinars; and is a member of the NSW Health Mental Health Review Tribunal. MT is a recipient of grant funding and commissions from the NHMRC, NSW Health, Sax Institute, Australian Government, Australian Rotary Health, Paul Ramsay Foundation and icare. She receives royalties from Oxford University Press for the sale of the adult COPE manual; receives honorarium and payment for lectures, seminars and webinars. She is a director of Climate Schools Pty Ltd, a company established to distribute evidence-based prevention resources to schools. SEB is a recipient of grant funding from the National Institutes of Health $(\mathrm{NIH})$, Department of Defense and Veterans Affairs; receives royalties from 0xford University Press for the sale of the adult COPE manual; and receives honorarium and payment for lectures and grant reviews. EB is a recipient of grant funding from NSW Health, NHMRC, Australian Rotary Health, Sax Institute and icare, and receives payment for lectures, seminars and webinars. VC is a recipient of grant funding from the NHMRC, Australian Rotary Health and Children's Hospital Foundation. She receives royalties from New Harbinger Press for the sale of a self-help book for parents of anxious children; and receives honorarium and payment for lectures, seminars and marking of theses. She is the first author of Fear-less Triple $\mathrm{P}$ and may in the future receive royalties from the dissemination of this resource. SB is a recipient of grant funding from the NHMRC, Movember Foundation, The Mental IIIness Research Fund, Australian Rotary Health and the Telstra Foundation. She receives honorarium and payment for lectures, seminars and workshops. SP is a recipient of grant funding from National Institutes for Health Research and charitable organisations in the UK and Sweden and receives income from books/book chapters and workshops on cognitivebehavioural therapy for paediatric post-traumatic stress disorder and anxiety disorders. JR is a recipient of grant funding from the NHMRC. KTB is a recipient of grant funding and commissions from the NIH, the Department of Defense and the Veterans Administration Medical System; receives royalties from American Psychiatric Press; receives honorarium and payment for lectures, seminars and webinars. NP is a recipient of grant funding from Australian Rotary Health. IK is a recipient of grant funding from Australian Rotary Health.

Patient consent for publication Not required.

Provenance and peer review Not commissioned; peer reviewed for ethical and funding approval prior to submission.

Supplemental material This content has been supplied by the author(s). It has not been vetted by BMJ Publishing Group Limited (BMJ) and may not have been peer-reviewed. Any opinions or recommendations discussed are solely those of the author(s) and are not endorsed by BMJ. BMJ disclaims all liability and responsibility arising from any reliance placed on the content. Where the content includes any translated material, BMJ does not warrant the accuracy and reliability of the translations (including but not limited to local regulations, clinical guidelines, terminology, drug names and drug dosages), and is not responsible for any error and/or omissions arising from translation and adaptation or otherwise.

Open access This is an open access article distributed in accordance with the Creative Commons Attribution Non Commercial (CC BY-NC 4.0) license, which permits others to distribute, remix, adapt, build upon this work non-commercially, and license their derivative works on different terms, provided the original work is properly cited, appropriate credit is given, any changes made indicated, and the use is non-commercial. See: http://creativecommons.org/licenses/by-nc/4.0/.

ORCID iD

Katherine L Mills http://orcid.org/0000-0002-9714-1832

\section{REFERENCES}

1 Barrett EL, Teesson M, Chapman C. Substance use and mental health consequences of childhood trauma: an epidemiological investigation. The College of problems of drug dependence scientific meeting. Puerto Rico, US, 2014.

2 Kessler RC, McLaughlin KA, Green JG, et al. Childhood adversities and adult psychopathology in the who world mental health surveys. British Journal of Psychiatry 2010;197:378-85.

3 Nooner KB, Linares LO, Batinjane J, et al. Factors related to posttraumatic stress disorder in adolescence. Trauma Violence Abuse 2012;13:153-66.

4 Brady KT, Back SE. Childhood trauma, posttraumatic stress disorder, and alcohol dependence. Alcohol Res 2012;34:408-13.

5 Khantzian EJ. The self-medication hypothesis of substance use disorders: a reconsideration and recent applications. Harv Rev Psychiatry 1997;4:231-44.

6 Perkonigg A, Kessler RC, Storz S, et al. Traumatic events and posttraumatic stress disorder in the community: prevalence, risk factors and comorbidity. Acta Psychiatr Scand 2000;101:46-59.

7 Wolitzky-Taylor K, Bobova L, Zinbarg RE, et al. Longitudinal investigation of the impact of anxiety and mood disorders in adolescence on subsequent substance use disorder onset and vice versa. Addict Behav 2012;37:982-5.

8 Giaconia RM, Reinherz HZ, Hauf AC, et al. Comorbidity of substance use and post-traumatic stress disorders in a community sample of adolescents. Am J Orthopsychiatry 2000;70:253-62.

9 Mills KL. 'Between pain and nothing, I choose nothing': trauma, post-traumatic stress disorder and substance use. Addiction 2009;104:1607-9.

10 Stewart SH, Conrod PJ. Psychological models of functional assocaitions between posttraumatic stress disorder and substance use disorder. In: Ouimette P, Brown PJ, eds. Trauma and substance abuse: causes, consequences, and treatment of disorders. Washington DC: American Psychological Association, 2003

11 Back SE, Brady KT, Jaanimägi U, et al. Cocaine dependence and PTSD: a pilot study of symptom interplay and treatment preferences. Addict Behav 2006;31:351-4.

12 Farrugia PL, Mills KL, Barrett E, et al. Childhood trauma among individuals with co-morbid substance use and post traumatic stress disorder. Ment Health Subst Use 2011;4:314-26.

13 De Bellis MD. The psychobiology of neglect. Child Maltreat 2005;10:150-72.

14 Marusak HA, Martin KR, Etkin A, et al. Childhood trauma exposure disrupts the automatic regulation of emotional processing. Neuropsychopharmacology 2015;40:1250-8.

15 Suarez LM, Belcher HME, Briggs EC, et al. Supporting the need for an integrated system of care for youth with co-occurring traumatic stress and substance abuse problems. Am J Community Psychol 2012;49:430-40.

16 Brown DW, Anda RF, Tiemeier $\mathrm{H}$, et al. Adverse childhood experiences and the risk of premature mortality. Am J Prev Med 2009;37:389-96.

17 Hall WD, Patton G, Stockings E, et al. Why young people's substance use matters for global health. Lancet Psychiatry 2016;3:265-79.

18 Chapman C, Mills K, Slade T, et al. Remission from posttraumatic stress disorder in the general population. Psychol Med 2012;42:1695-703.

19 Gerson R, Rappaport N. Traumatic stress and posttraumatic stress disorder in youth: recent research findings on clinical impact, assessment, and treatment. J Adolesc Health 2013;52:137-43. 
20 Bradizza CM, Stasiewicz PR, Paas ND. Relapse to alcohol and drug use among individuals diagnosed with co-occurring mental health and substance use disorders: a review. Clin Psychol Rev 2006;26:162-78.

21 Back SE, Brady KT, Sonne SC, et al. Symptom improvement in co-occurring PTSD and alcohol dependence. J Nerv Ment Dis 2006;194:690-6.

22 Cohen JA, Mannarino AP, Zhitova AC, et al. Treating child abuserelated posttraumatic stress and comorbid substance abuse in adolescents. Child Abuse Negl 2003;27:1345-65.

23 Mills KL, Teesson M, Back SE, et al. Integrated exposure-based therapy for co-occurring posttraumatic stress disorder and substance dependence: a randomized controlled trial. JAMA 2012;308:690-9.

24 Danielson CK, McCart MR, Walsh K, et al. Reducing substance use risk and mental health problems among sexually assaulted adolescents: a pilot randomized controlled trial. J Fam Psychol 2012;26:628-35.

25 Fortuna LR, Porche MV, Padilla A. A treatment development study of a cognitive and mindfulness-based therapy for adolescents with cooccurring post-traumatic stress and substance use disorder. Psychol Psychother 2018;91:42-62.

26 Kmett Danielson C, McCart MR, de Arellano MA, et al. Risk reduction for substance use and trauma-related psychopathology in adolescent sexual assault victims: findings from an open trial. Child Maltreat 2010;15:261-8.

27 Najavits LM, Gallop RJ, Weiss RD. Seeking safety therapy for adolescent girls with PTSD and substance use disorder: a randomized controlled trial. J Behav Health Serv Res 2006;33:453-63.

28 Danielson CK, Adams Z, McCart MR, et al. Safety and efficacy of Exposure-Based risk reduction through family therapy for cooccurring substance use problems and posttraumatic stress disorder symptoms among adolescents: a randomized clinical trial. JAMA Psychiatry 2020;77:574-86.

29 Roberts NP, Roberts PA, Jones N, et al. Psychological interventions for post-traumatic stress disorder and comorbid substance use disorder: a systematic review and meta-analysis. Clin Psychol Rev 2015;38:25-38.

30 Back SE, Foa EB, Killeen TK, et al. Brady K. T. concurrent treatment of PTSD and substance use disorders using prolonged exposure (cope) therapist manual. NY: Oxford Univeristy Press, 2015.

31 Ruglass LM, Lopez-Castro T, Papini S, et al. Concurrent treatment with prolonged exposure for co-occurring full or subthreshold posttraumatic stress disorder and substance use disorders: a randomized clinical trial. Psychother Psychosom 2017;86:150-61.

32 Back SE, Killeen T, Badour CL, et al. Concurrent treatment of substance use disorders and PTSD using prolonged exposure: a randomized clinical trial in military veterans. Addict Behav 2019;90:369-77.

33 Waschbusch DA, Fabiano G, Pelham Jr. WE. Evidence-based practice in child and adolescent disorders. In: Sturmey PH M, ed. Handbook of evidence-based practice in clinical psychology. Wiley, 2012.

34 Foa EB, Chrestman KR, Gilboa-Schechtman E. Prolonged exposure therapy for adolescents with PTSD: emotional processing of traumatic experiences. New York: Oxford University Press, 2009.

35 Knight JR, Shrier LA, Bravender TD, et al. A new brief screen for adolescent substance abuse. Arch Pediatr Adolesc Med 1999;153:591-6.

36 Pynoos RS, Steinberg AM. The University of California, Los Angeles, post-traumatic stress disorder reaction index (UCLA PTSD index) for the diagnostic and statistical manual of mental disorders. 5th ed. Los Angeles: University of California, 2015.

37 Sheehan DV, Sheehan KH, Shytle RD, et al. Reliability and validity of the mini international neuropsychiatric interview for children and adolescents (MINI-KID). J Clin Psychiatry 2010;71:313-26.

38 Ehlers A, Bisson J, Clark DM, et al. Do all psychological treatments really work the same in posttraumatic stress disorder? Clin Psychol Rev 2010;30:269-76.

39 Kay-Lambkin FJ, Baker AL, Kelly B, et al. Clinician-assisted computerised versus therapist-delivered treatment for depressive and addictive disorders: a randomised controlled trial. Med J Aust 2011;195:S44-50.

40 Pynoos R W FW, Steinberg AM, Marx BP, et al. ClinicianAdministered PTSD Scale for DSM-5 - Child/Adolescent Version, 2015. Available: www.ptsd.va.gov
41 Sobell L S MB. Timeline Follow-Back: A Technique for Assessing Self-Reported Alcohol Consumption. In: RZLaJP A, ed. Measuring alcohol consumption: psychosocial and biochemical methods. Totowa, NJ: Humana Press, 1992: 41-72.

42 Fisher PW. Diagnostic interview schedule for children, version 5 (DISC-5. New York, NY: Child \& Adolescent Psychiatry, New York State Psychiatric Institute/Columbia University - Department of Psychiatry, 2020.

43 Breslin FC, Sobell LC, Sobell MB, et al. A comparison of a brief and long version of the situational confidence questionnaire. Behav Res Ther 2000;38:1211-20.

44 Meiser-Stedman R, Smith P, Bryant R, et al. Development and validation of the child post-traumatic Cognitions inventory (CPTCI). J Child Psychol Psychiatry 2009;50:432-40.

45 Varni JW, Seid M, Rode CA. The PedsQL TM: measurement model for the pediatric quality of life inventory. Med Care 1999;37:126-39.

46 Chorpita BF, Yim L, Moffitt C, et al. Assessment of symptoms of DSM-IV anxiety and depression in children: a revised child anxiety and depression scale. Behav Res Ther 2000;38:835-55.

47 Gullone E, Taffe J. The emotion regulation questionnaire for children and adolescents (ERQ-CA): a psychometric evaluation. Psychol Assess 2012;24:409-17.

48 Bijttebier P, Raes F, Vasey MW, et al. Assessment of repetitive negative thinking in children: the perseverative thinking questionnaire - child version (PTQ-C). J Psychopathol Behav Assess 2015;37:164-70.

49 Neff KD, McGehee P. Self-compassion and psychological resilience among adolescents and young adults. Self and Identity 2010:9:225-40.

50 Goodman R. A modified version of the Rutter parent questionnaire including extra items on children's strengths: a research note. J Child Psychol Psychiatry 1994;35:1483-94.

51 Hafekost J, Lawrence D, Boterhoven de Haan K, et al. Methodology of young minds matter: the second Australian child and adolescent survey of mental health and wellbeing. Aust $N Z J$ Psychiatry 2016;50:866-75.

52 Darke S, Hall W, Wodak A, et al. Development and validation of a multi-dimensional instrument for assessing outcome of treatment among opiate users: the opiate treatment index. $\mathrm{Br} J$ Addict 1992:87:733-42.

53 Goldschmidt AB, Doyle AC, Wilfley DE. Assessment of binge eating in overweight youth using a questionnaire version of the child eating disorder examination with instructions. Int $J$ Eat Disord 2007;40:460-7.

54 Epstein NB, Baldwin LM, Bishop DS. The McMaster family assessment DEVICE*. J Marital Fam Ther 1983;9:171-80.

55 Shapiro JP, Welker CJ, Jacobson BJ. The youth client satisfaction questionnaire: development, construct validation, and factor structure. J Clin Child Psychol 1997;26:87-98.

56 Attkisson CC, Zwick R. The client satisfaction questionnaire. psychometric properties and correlations with service utilization and psychotherapy outcome. Eval Program Plann 1982;5:233-7.

57 Osman A, Bagge CL, Gutierrez PM, et al. The suicidal behaviors Questionnaire-Revised (SBQ-R): validation with clinical and nonclinical samples. Assessment 2001;8:443-54.

58 Hatcher RL, Gillaspy JA. Development and validation of a revised short version of the working alliance inventory. Psychotherapy Research 2006;16:12-25.

59 Grant VV, Stewart SH, O'Connor RM, et al. Psychometric evaluation of the five-factor Modified Drinking Motives Questionnaire--Revised in undergraduates. Addict Behav 2007:32:2611-32.

60 Dube SR, Anda RF, Felitti VJ, et al. Childhood abuse, household dysfunction, and the risk of attempted suicide throughout the life span: findings from the adverse childhood experiences study. JAMA 2001;286:3089-96.

61 Varni JW, Seid M, Rode CA. The PedsQL: measurement model for the pediatric quality of life inventory. Med Care 1999;37:126-39.

62 Achenbach T, Rescorla LA. Manual for the ASEBA School-Age Forms \& Profiles. Burlington, VT: University of Vermont, Research Center for Children, Youth, and Families, 2001.

63 Olweus D. Olweus bullying questionnaire. Center City: Hazelden, 2007.

64 Gosling SD, Rentfrow PJ, Swann WB. A very brief measure of the Big-Five personality domains. J Res Pers 2003;37:504-28.

65 Simons JS, Gaher RM. The distress tolerance scale: development and validation of a self-report measure. Motiv Emot 2005;29:83-102. 